\title{
A NOTE ON THE BOUNDARY REPRESENTATION OF A CONTINUOUS SPATIAL SEMIGROUP OF *-ENDOMORPHISMS OF $\mathscr{B}(\mathscr{H})$
}

\author{
ALEXIS ALEVRAS
}

(Communicated by Palle E. T. Jorgensen)

\begin{abstract}
We prove that the equivalence class of the boundary representation $\pi_{\alpha, \mathscr{L}}$ of an $E_{0}$-semigroup $\alpha$ is independent of the intertwining semigroup of isometries $\mathscr{U}$.
\end{abstract}

\section{INTRODUCTION}

An $E_{0}$-semigroup of $\mathscr{B}(\mathscr{H})$, where $\mathscr{H}$ is a separable Hilbert space, is a $\sigma$ weakly continuous semigroup $\alpha=\left\{\alpha_{t}: t \geq 0\right\}$ of unit-preserving *-endomorphisms of $\mathscr{B}(\mathscr{H})$ with $\alpha_{0}=\mathrm{id}_{\mathscr{B}(\mathscr{H})}$. In [3] Powers introduced a numerical index for spatial $E_{0}$-semigroups, i.e. for $E_{0}$-semigroups $\alpha$ for which there is a strongly continuous semigroup of isometries $\mathscr{U}=\left\{U_{t}: t \geq 0\right\}$ of $\mathscr{B}(\mathscr{H})$ that intertwines $\alpha$, in the sense that $\alpha_{t}(A) U_{t}=U_{t} A$ for all $A \in \mathscr{B}(\mathscr{H})$ and all $t \geq 0$. Starting with an $E_{0}$-semigroup $\alpha$ and an intertwining semigroup of isometries $\mathscr{U}$, he defined a continuous $*$-representation, $\pi_{\alpha}, \mathscr{U}$, of the domain of definition $\mathscr{D}(\delta)$ of the generator $\delta$ of $\alpha$, on the deficiency space of $\mathscr{U}$. The index was then defined to be the multiplicity of $\pi_{\alpha, \mathscr{U}}$, i.e. the maximum number of mutually orthogonal projections that commute with the range of $\pi_{\alpha, \mathscr{U}}$. However, since an intertwining semigroup of isomeiries, if it exists, need not be unique, and since there is no canonical way of selecting one, it is not clear that this numerical index is well defined.

In [5] Powers and Price discovered the relation between the boundary representation $\pi_{\alpha, \mathscr{U}}$ and the Arveson index of an $E_{0}$-semigroup $\alpha$ (see [1] for the definition of the Arveson index, or [2] for an expository account of Arveson's theory of $E_{0}$-semigroups). They proved that the Arveson index of a spatial $E_{0^{-}}$ semigroup is equal to the multiplicity of the identity representation of $\mathscr{D}(\delta)$ in the representation $\pi_{\alpha, \mathscr{U}}$. It then follows of course, that this multiplicity is independent of the choice of $\mathscr{U}$. However it is not known whether the multiplicity of the identity representation of $\mathscr{D}(\delta)$ in $\pi_{\alpha, \mathscr{U}}$ is equal to the multiplicity of $\pi_{\alpha, \mathscr{U}}$ as defined above, i.e., it is not known whether $\pi_{\alpha, \mathscr{U}}$ is always normal. In this note we establish the fact that the multiplicity of $\pi_{\alpha, \mathscr{U}}$ does not depend

Received by the editors March 29, 1994.

1991 Mathematics Subject Classification. Primary 46L40; Secondary 46L55, 46L30. 
on the choice of the intertwining semigroup of isometries. More specifically we shall prove the following:

Theorem. Let $\alpha$ be an $E_{0}$-semigroup of $\mathscr{B}(\mathscr{H})$, and suppose that $\mathscr{U}$ and $\mathscr{S}$ are two strongly continuous intertwining semigroups of isometries. Then the two boundary representations $\pi_{\alpha, \mathscr{U}}$ and $\pi_{\alpha, \mathscr{S}}$ are unitarily equivalent.

It then follows that the multiplicity of $\pi_{\alpha, \mathscr{U}}$ is a well-defined index, which is subadditive and invariant under inner conjugacy (i.e., under perturbation of the generator by an inner derivation). Finally we note that this index is equal to the Arveson index for all known examples of spatial $E_{0}$-semigroups. The equality of the two indices in the general case is equivalent to the normality of $\pi_{\alpha, \mathscr{U}}$.

\section{Preliminaries: The boundary Representation}

Definition. An $E_{0}$-semigroup of $\mathscr{B}(\mathscr{H})$ is a family $\alpha=\left\{\alpha_{t}: t \geq 0\right\}$ such that:

(i) $\alpha_{t}$ is a normal $*$-endomorphism of $\mathscr{B}(\mathscr{H})$ for every $t \geq 0$;

(ii) $\alpha_{0}=\operatorname{id}_{\mathscr{B}(\mathscr{H})}$ and $\alpha_{t+s}=\alpha_{t} \circ \alpha_{s}$ for all $s, t \geq 0$;

(iii) for each $f \in \mathscr{B}(\mathscr{H})_{*}$ and for each $A \in \mathscr{B}(\mathscr{C})$ the function $t \rightarrow$ $f\left(\alpha_{t}(A)\right), t \geq 0$, is continuous;

(iv) $\alpha_{t}(1)=1$ for all $t \geq 0$.

Definition. An intertwining semigroup of isometries for an $E_{0}$-semigroup $\alpha$ of $\mathscr{B}(\mathscr{H})$ is a strongly continuous semigroup of isometries, $\mathscr{U}=\left\{U_{t}: t \geq 0\right\}$, such that:

$$
\alpha_{t}(A) U_{t}=U_{t} A \text { for all } t \geq 0 \text { and } A \in \mathscr{B}(\mathscr{H}) .
$$

In [4] Powers constructed an example of an $E_{0}$-semigroup of $\mathscr{B}(\mathscr{H})$ which does not have an intertwining semigroup of isometries. If the $E_{0}$-semigroup $\alpha$ does have an intertwining semigroup of isometries, $\mathscr{U}$, then one can associate with it a $*$-representation $\pi_{\alpha, \mathscr{U}}$ of a certain $C^{*}$-algebra as follows:

Let $-d$ be the generator of $\mathscr{U}$ (i.e., $d$ is the operator with domain $\mathscr{D}(d)=$ $\left\{\xi \in \mathscr{H}: \lim _{t \rightarrow 0+} \frac{\xi-U_{t} \xi}{t}\right.$ exists $\}$ and for $\left.\xi \in \mathscr{D}(d), d \xi=\lim _{t \rightarrow 0+} \frac{\xi-U_{t} \xi}{t}\right)$. Then $d$ is a closed densely defined maximal skew-symmetric operator. For $\xi, \eta \in$ $\mathscr{D}\left(d^{*}\right)$ define

$$
\langle\xi, \eta\rangle_{*}=\frac{1}{2}\left\langle d^{*} \xi, \eta\right\rangle+\frac{1}{2}\left\langle\xi, d^{*} \eta\right\rangle
$$

where $\langle\cdot, \cdot\rangle$ is the inner product of $\mathscr{H}$. Then $\langle\cdot, \cdot\rangle_{*}$ is a positive semidefinite inner product, with null space $\mathscr{D}(d)$. Thus it induces an inner product on $\mathscr{D}\left(d^{*}\right) / \mathscr{D}(d)$ with which the latter becomes a Hilbert space. We denote the class of $\xi \in \mathscr{D}\left(d^{*}\right)$ in $\mathscr{D}\left(d^{*}\right) / \mathscr{D}(d)$ by $[\xi]$.

Consider now the generator $\delta$ of $\alpha$. The domain of $\delta$ is $\mathscr{D}(\delta)=\{A \in$ $\mathscr{B}(\mathscr{H}): s-\lim _{t \rightarrow 0+} \frac{\alpha_{t}(A)-A}{t}$ exists $\}$ and for $A \in \mathscr{D}(\delta), \delta(A)=s-\lim _{t \rightarrow 0+} \frac{\alpha_{t}(A)-A}{t}$. $\mathscr{D}(\delta)$ is a $\sigma$-weakly dense $*$-subalgebra of $\mathscr{B}(\mathscr{H})$ and $\delta$ is a $\sigma$-weakly closed *-derivation. Define

$$
\pi_{\alpha, \mathscr{U}}: \mathscr{D}(\delta) \rightarrow \mathscr{B}\left(\mathscr{D}\left(d^{*}\right) / \mathscr{D}(d)\right)
$$

by

$$
\pi_{\alpha, \mathscr{U}}(x)[\xi]=[x \xi] \text { for } x \in \mathscr{D}(\delta) \text { and } \xi \in \mathscr{D}\left(d^{*}\right)
$$


Proposition (Powers [3]). $\pi_{\alpha, \mathscr{U}}$ is a norm-continuous $*$-representation of $\mathscr{D}(\delta)$ (and therefore extends to a $*$-representation of the norm closure of $\mathscr{D}(\delta)$ ).

$\pi_{\alpha, \mathscr{U}}$ is called the boundary representation associated with the $E_{0}$-semigroup $\alpha$ and its intertwining semigroup $\mathscr{U}$.

\section{Proof of THE THEOREM}

The proof of the theorem depends on the analysis of pairs of semigroups of isometries $(\mathscr{S}, \mathscr{U})$ satisfying the commutation relations $S_{t}^{*} U_{t}=e^{-\lambda t} I$ for some $\lambda \in \mathbb{R}_{+}$, carried out by Powers and Price in [5]. We are making use of the following result:

Theorem (Powers and Price [5]). Let $\alpha$ be an $E_{0}$-semigroup with generator $\delta, \mathscr{U}$ an intertwining semigroup of isometries with generator $-d$ and $\pi_{\alpha, \mathscr{U}}$ the associated boundary representation of $\mathscr{D}(\delta)$ on $\mathscr{D}\left(d^{*}\right) / \mathscr{D}(d)$. If $\mathscr{S}$ is a strongly continuous semigroup of isometries with generator $-D$ such that $S_{t}^{*} U_{t}=$ $e^{-\lambda t}$ for some $\lambda>0$ and all $t \geq 0$, then:

(i) there is an isometry $W: \mathscr{H} \rightarrow \mathscr{D}\left(d^{*}\right) / \mathscr{D}(d)$ such that

$$
\mathscr{D}(D)=\left\{\xi \in \mathscr{D}\left(d^{*}\right): W \xi=\frac{1}{\sqrt{\lambda}}[\xi]\right\}
$$

(ii) $\mathscr{D}\left(D^{*}\right)=\mathscr{D}\left(d^{*}\right)$ and for $\xi \in \mathscr{D}\left(D^{*}\right), D^{*} \xi=d^{*} \xi+\lambda \xi-2 \sqrt{\lambda} W^{*}[\xi]$;

(iii) $\mathscr{S}$ intertwines $\alpha$ if and only if $W$ intertwines $\pi_{\alpha, \mathscr{U}}$ with $\mathrm{id}_{\mathscr{D}(\delta)}$, the identity representation of $\mathscr{D}(\boldsymbol{\delta})$.

We now state and prove the result of this note:

Theorem. Let $\alpha$ be an $E_{0}$-semigroup of $\mathscr{B}(\mathscr{H})$, and suppose that $\mathscr{U}$ and $\mathscr{S}$ are two strongly continuous intertwining semigroups of isometries. Then the two boundary representations $\pi_{\alpha, \mathscr{U}}$ and $\pi_{\alpha, \mathscr{S}}$ are unitarily equivalent.

PRoof. By ([1], Theorem 4.1), there is a complex number $c(\mathscr{U}, \mathscr{S})$, the covariance of $\mathscr{U}$ and $\mathscr{S}$, such that $S_{t}^{*} U_{t}=e^{c(\mathscr{U}, \mathscr{S}) t} I \quad \forall t \geq 0$. We first assume that the covariance is real, i.e., we assume that $S_{t}^{*} U_{t}=e^{-\lambda t} I \quad \forall t \geq 0$, for some $\lambda>0$.

Let $\delta$ be the generator of $\alpha$ and $-d_{\mathscr{U}}$ (resp. $-d_{\mathscr{S}}$ ) the generator of $\mathscr{U}$ (resp. of $\mathscr{S})$. Let $\mathscr{K}_{\mathscr{U}}=\mathscr{D}\left(d_{\mathscr{Q}}^{*}\right) / \mathscr{D}\left(d_{\mathscr{U}}\right)$ be the Hilbert space with inner product given by

$$
\langle[\xi],[\eta]\rangle_{\mathscr{L}}=\frac{1}{2}\left\langle d_{\mathscr{L}}^{*} \xi, \eta\right\rangle+\frac{1}{2}\left\langle\xi, d_{\mathscr{L}}^{*} \eta\right\rangle
$$

where [ $\xi]$ denotes the class in $\mathscr{D}\left(d_{\mathscr{U}}^{*}\right) / \mathscr{D}\left(d_{\mathscr{U}}\right)$ of an element $\xi \in \mathscr{D}\left(d_{\mathscr{U}}^{*}\right)$. Similarly let $\mathscr{K}_{\mathscr{S}}=\mathscr{D}\left(d_{\mathscr{S}}^{*}\right) / \mathscr{D}\left(d_{\mathscr{S}}\right)$ be the Hilbert space with inner product

$$
\langle\{\xi\},\{\eta\}\rangle_{\mathscr{S}}=\frac{1}{2}\left\langle d_{\mathscr{S}}^{*} \xi, \eta\right\rangle+\frac{1}{2}\left\langle\xi, d_{\mathscr{S}}^{*} \eta\right\rangle
$$

where $\{\xi\}$ denotes the class in $\mathscr{D}\left(d_{\mathscr{S}}^{*}\right) / \mathscr{D}\left(d_{\mathscr{S}}\right)$ of an element $\xi \in \mathscr{D}\left(d_{\mathscr{S}}^{*}\right)$.

By the previous theorem we have $\mathscr{D}\left(d_{\mathscr{S}}^{*}\right)=\mathscr{D}\left(d_{\mathscr{U}}^{*}\right)$ and there is an isometry $W_{\mathscr{U}}: \mathscr{H} \rightarrow \mathscr{K}_{\mathscr{U}}$ that intertwines $\pi_{\alpha, \mathscr{U}}$ with id $\operatorname{do}_{\mathscr{D}(\delta)}$, such that $\mathscr{D}\left(d_{\mathscr{S}}\right)=\{\xi \in$ $\left.\mathscr{D}\left(d_{\mathscr{U}}^{*}\right): W_{\mathscr{L}} \xi=\frac{1}{\sqrt{\lambda}}[\xi]\right\}$. Since we also have $U_{t}^{*} S_{t}=e^{-\lambda t} I \quad \forall t \geq 0$, there is, by the same theorem, an isometry $W_{\mathscr{S}}: \mathscr{H} \rightarrow \mathscr{K}_{\mathscr{S}}$ that intertwines $\pi_{\alpha, \mathscr{S}}$ with 
$\operatorname{id}_{\mathscr{D}(\delta)}$, such that $\mathscr{D}\left(d_{\mathscr{S}}\right)=\left\{\xi \in \mathscr{D}\left(d_{\mathscr{S}}^{*}\right): W_{\mathscr{S}} \xi=\frac{1}{\sqrt{\lambda}}\{\xi\}\right\}$. Moreover we have the following relations between $d_{\mathscr{L}}^{*}$ and $d_{\mathscr{S}}^{*}$ :

$$
d_{\mathscr{S}}^{*} \xi=d_{\mathscr{U}}^{*} \xi+\lambda \xi-2 \sqrt{\lambda} W_{\mathscr{U}}^{*}[\xi] \text { and } d_{\mathscr{L}}^{*} \xi=d_{\mathscr{S}}^{*} \xi+\lambda \xi-2 \sqrt{\lambda} W_{\mathscr{S}}^{*}\{\xi\}
$$

for $\xi \in \mathscr{D}\left(d_{\mathscr{U}}^{*}\right)=\mathscr{D}\left(d_{\mathscr{S}}^{*}\right)$.

Define $V: \mathscr{K}_{\mathscr{S}} \rightarrow \mathscr{K}_{\mathscr{U}}$ by

$$
V(\{\xi\})=[\xi]-\sqrt{\lambda} W_{\mathscr{U}} \xi
$$

We claim that $V$ is a (well-defined) unitary operator that intertwines $\pi_{\alpha, \mathscr{U}}$ and $\pi_{\alpha, \mathscr{S}}$.

If $\{\xi\}=\{\eta\}, \xi, \eta \in \mathscr{D}\left(d_{\mathscr{S}}^{*}\right)$, then $\xi-\eta \in \mathscr{D}\left(d_{\mathscr{S}}\right)$ and, therefore, $W_{\mathscr{U}}(\xi-\eta)=\frac{1}{\sqrt{\lambda}}[\xi-\eta]$, by (i) of the above proposition. This implies that $[\xi]-\sqrt{\lambda} W_{\mathscr{U}} \xi=[\eta]-\sqrt{\lambda} W_{\mathscr{U}} \eta \Leftrightarrow V(\{\xi\})=V(\{\eta\})$. Thus $V$ is well defined.

To prove that $V$ is isometric let $\xi, \eta \in \mathscr{D}\left(d_{\mathscr{S}}^{*}\right)$, and calculate

$$
\begin{aligned}
\langle V(\{\xi\}), V(\{\eta\})\rangle_{\mathscr{U}}= & \left\langle[\xi]-\sqrt{\lambda} W_{\mathscr{U}} \xi,[\eta]-\sqrt{\lambda} W_{\mathscr{U}} \eta\right\rangle_{\mathscr{U}} \\
= & \langle[\xi],[\eta]\rangle_{\mathscr{C}}+\lambda\left\langle W_{\mathscr{U}} \xi, W_{\mathscr{U}} \eta\right\rangle_{\mathscr{U}} \\
& -\sqrt{\lambda}\left\langle W_{\mathscr{U}} \xi,[\eta]\right\rangle_{\mathscr{U}}-\sqrt{\lambda}\left\langle[\xi], W_{\mathscr{U}} \eta\right\rangle_{\mathscr{U}} \\
= & \langle[\xi],[\eta]\rangle_{\mathscr{U}}+\lambda\langle\xi, \eta\rangle-\sqrt{\lambda}\left\langle W_{\mathscr{U}} \xi,[\eta]\right\rangle_{\mathscr{U}}-\sqrt{\lambda}\left\langle[\xi], W_{\mathscr{U}} \eta\right\rangle_{\mathscr{U}}
\end{aligned}
$$

using the fact that $W_{\mathscr{U}}$ is isometric. On the other hand,

$$
\begin{aligned}
\langle\{\xi\},\{\eta\}\rangle_{\mathscr{S}}= & \frac{1}{2}\left\langle d_{\mathscr{S}}^{*} \xi, \eta\right\rangle+\frac{1}{2}\left\langle\xi, d_{\mathscr{S}}^{*} \eta\right\rangle \\
= & \frac{1}{2}\left\langle d_{\mathscr{U}}^{*} \xi+\lambda \xi-2 \sqrt{\lambda} W_{\mathscr{U}}^{*}[\xi], \eta\right\rangle+\frac{1}{2}\left\langle\xi, d_{\mathscr{U}}^{*} \eta+\lambda \eta-2 \sqrt{\lambda} W_{\mathscr{U}}^{*}[\eta]\right\rangle \\
= & \frac{1}{2}\left\langle d_{\mathscr{U}}^{*} \xi, \eta\right\rangle+\frac{1}{2}\left\langle\xi, d_{\mathscr{U}}^{*} \eta\right\rangle+\lambda\langle\xi, \eta\rangle \\
& -\sqrt{\lambda}\left\langle W_{\mathscr{U}}^{*}[\xi], \eta\right\rangle-\sqrt{\lambda}\left\langle\xi, W_{\mathscr{U}}^{*}[\eta]\right\rangle \\
= & \langle[\xi],[\eta]\rangle_{\mathscr{U}}+\lambda\langle\xi, \eta\rangle-\sqrt{\lambda}\left\langle[\xi], W_{\mathscr{U}} \eta\right\rangle_{\mathscr{U}}-\sqrt{\lambda}\left\langle W_{\mathscr{U}} \xi,[\eta]\right\rangle_{\mathscr{U}}
\end{aligned}
$$

and therefore $V$ is isometric.

Next define $V_{1}: \mathscr{K}_{\mathscr{Q}} \rightarrow \mathscr{K}_{\mathscr{S}}$ by

$$
V_{1}([\xi])=\{\xi\}-\sqrt{\lambda} W_{\mathscr{S}} \xi .
$$

$V_{1}$ is a well-defined isometric operator. For $\xi \in \mathscr{D}\left(d_{\mathscr{\ell}}^{*}\right)=\mathscr{D}\left(d_{\mathscr{S}}^{*}\right)$ we have

$$
\begin{aligned}
V V_{1}([\xi]) & =V(\{\xi\})-\sqrt{\lambda} V W_{\mathscr{S}} \xi \\
& =[\xi]-\sqrt{\lambda} W_{\mathscr{L}} \xi-\sqrt{\lambda} V W_{\mathscr{S}} \xi \\
& =[\xi]-\sqrt{\lambda}\left(W_{\mathscr{U}} \xi+V W_{\mathscr{S}} \xi\right) .
\end{aligned}
$$

Considering a sequence $\left(\xi_{n}\right)$ in $\mathscr{D}\left(d_{\mathscr{U}}\right)$ that converges to $\xi$ we find

$$
\begin{aligned}
W_{\mathscr{U}} \xi+V W_{\mathscr{S}} \xi & =W_{\mathscr{U}} \xi+\lim V W_{\mathscr{S}} \xi_{n} \\
& =W_{\mathscr{U}} \xi+\lim V\left(\frac{1}{\sqrt{\lambda}}\left\{\xi_{n}\right\}\right) \\
& =W_{\mathscr{U}} \xi+\lim \frac{1}{\sqrt{\lambda}}\left(\left[\xi_{n}\right]-\sqrt{\lambda} W_{\mathscr{U}} \xi_{n}\right) \\
& =W_{\mathscr{U}} \xi-\lim W_{\mathscr{U}} \xi_{n} \\
& =0 .
\end{aligned}
$$


It follows that $V V_{1}$ is the identity on $\mathscr{K}_{\mathscr{S}}$ and therefore that $V$ is a unitary operator. We finally prove that $V$ intertwines $\pi_{\alpha, \mathscr{U}}$ and $\pi_{\alpha, \mathscr{S}}$. For $A \in \mathscr{D}(\delta)$ and $\xi \in \mathscr{D}\left(d_{\mathscr{\ell}}^{*}\right)=\mathscr{D}\left(d_{\mathscr{S}}^{*}\right)$ we have

$$
\begin{aligned}
\pi_{\alpha, \mathscr{U}}(A) V(\{\xi\}) & =\pi_{\alpha, \mathscr{U}}(A)\left([\xi]-\sqrt{\lambda} W_{\mathscr{U}} \xi\right) \\
& =[A \xi]-\sqrt{\lambda} \pi_{\alpha, \mathscr{U}}(A) W_{\mathscr{U}} \xi \\
& =[A \xi]-\sqrt{\lambda} W_{\mathscr{U}} A \xi \\
& =V(\{A \xi\}) \\
& =V \pi_{\alpha, \mathscr{S}}(A)(\{\xi\})
\end{aligned}
$$

using the fact that $W_{\mathscr{U}}$ intertwines $\pi_{\alpha, \mathscr{U}}$ with the identity representation of $\mathscr{D}(\delta)$.

If now $\mathscr{U}$ and $\mathscr{S}$ are two intertwining semigroups of isometries with covariance $c(\mathscr{U}, \mathscr{S}) \in \mathbb{C}$, then consider the intertwining semigroup

$$
\mathscr{S}^{\prime}=\left\{e^{-i \operatorname{Im}(c(\mathscr{U}, \mathscr{S})) t} S_{t}: t \geq 0\right\} .
$$

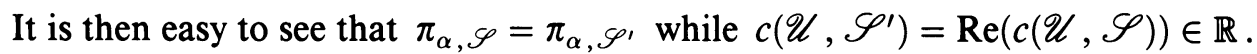
From the first part of the proof, it follows that $\pi_{\alpha, \mathscr{U}}$ and $\pi_{\alpha, \mathscr{S}}$ are unitarily equivalent. This completes the proof.

\section{REFERENCES}

1. W. Arveson, Continuous analogues of Fock space, Mem. Amer. Math. Soc. 80409 (1989).

2. ___, Quantizing the Fredholm index, Proc. of the Great Plains Operator Theory Seminar, Pitman Res. Notes Math. Ser., vol. 225, Longman Sci. Tech., Harlow, 1988.

3. R. T. Powers, An index theory for semigroups of *-endomorphisms of $\mathscr{B}(\mathscr{H})$ and type $\mathrm{II}_{1}$ factors, Canad. J. Math. 40 (1988), 86-114.

4. $\_$, A non spatial continuous semigroup of $*$-endomorphisms of $\mathscr{B}(\mathscr{H})$, Publ. Res. Inst. Math. Sci. 23 (1987), 1053-1069.

5. R. T. Powers and G. Price, Continuous spatial semigroups of $*$-endomorphisms of $\mathscr{B}(\mathscr{H})$, Trans. Amer. Math. Soc. 321 (1990), 347-361.

Department of Mathematics, University of California, Berkeley, California 94720

E-mail address: alevras@math.berkeley .edu 